\title{
Mechanism of Concentration-Dependent Vesicle-Micelle Transition in Aqueous Mixture of Cationic and Anionic Surfactants
}

\author{
Hisanori Nakanishi ${ }^{1}$, Koji Tsuchiya ${ }^{2}$, Takahiro Ohкubo ${ }^{2}$, Hideki SaKai ${ }^{1,2}$ and Masahiko Abe ${ }^{1 *, 2}$ \\ ${ }^{I}$ Faculty of Science and Technology, Tokyo University of Science \\ (Yamazaki 2641, Noda, Chiba 278-8510, JAPAN) \\ ${ }^{2}$ Institute of Colloid and Interface Science, Tokyo University of Science \\ (Kagurazaka 1-3, Shinjuku, Tokyo 162-0825, JAPAN)
}

Edited by H. Kunieda, Yokohama Nat. Univ., and accepted April 15, 2005 (received for review March 22, 2005)

\begin{abstract}
The phase behavior of aqueous mixed solution of cationic cetyltrimethylammonium bromide (CTAB) and anionic sodium octylsulfate was studied focusing on the concentration-dependent vesicle-micelle transition. Dynamic light scattering measurements and freeze fracture TEM observations showed that the phase transition occurs in two steps depending on the total surfactant concentration. Equilibrium vesicles $(V)$ formed in dilute solution was shown to transform to micelles $(M)$ through a micelle/ small unilamellar vesicle $(M+V)$ coexisting state. The concentration at which the transition from V to $\mathrm{M}+\mathrm{V}$ occurs on the SOSrich side lies around the CMC of pure SOS $(120 \mathrm{mM})$. Moreover, electroconductivity and FT-IR measurements revealed that the phase transition is accompanied by a decrease in the degree of counter ion dissociation from surfactant molecules. Based on these experimental findings, the cause of the phase transition was discussed.
\end{abstract}

Key words: mixed surfactant system, phase behavior, spontaneous vesicle, micelle, FFTEM, electroconductivity, FT- IR

\section{Introduction}

Mixtures of surfactants in aqueous solution exhibit different levels of synergism depending on the charge and molecular structure of individual surfactant components (1). In particular, mixtures of cationic and anionic surfactants provide a rich source of phase behavior in aqueous solutions. Thus, spherical and rod-like micelles, vesicles, lamellae and precipitates are observed depending on the total surfactant concentration and the mixing ratio of the two surfactants. These properties of the mixtures can be exploited in many applications. For example, rod-like micelles have found their uses as a drag-reduction agent in pipeline flow (2) and vesicles are expected to be useful as a carrier in drug delivery systems (DDS) (3), a new organic reac- tion field (4) and a biomembrane model (5).

This unusual phase behavior of the mixture was ascribed to anionic-cationic surfactant complexes formed through the electrostatic attraction between the charges on the ionic headgroups (6). The area occupied by the headgroups in the complex was less than the sum of the areas occupied by the head groups of two ionic surfactants in solutions because of the electrostatic interaction. This is the reason why the CAC (Critical Aggregation Concentration) of the complex was much lower than the $\mathrm{CMC}$ of either of the anionic and cationic surfactant components $(7,8)$. Furthermore, the complexes often form vesicles spontaneously (9-11).

In general, while vesicles often form spontaneously in solutions of single-component surfactants or amphipathic substances in vivo, they do not form in vitro.

\footnotetext{
${ }^{*}$ Correspondence to: Masahiko ABE, Faculty of Science and Technology, Tokyo University of Science, 2641 Yamazaki, Noda, Chiba 2788510, JAPAN

E-mail: abemasa@rs.noda.tus.ac.jp
} 
Preparation of unilamellar vesicles (liposomes) is usually made by mechanically disrupting a dispersion of lamellar liquid crystalline phase with sonication or elaborating chemical treatments. The vesicles thus prepared are normally metastable and gradually revert to lamellar liquid crystalline phase (3). Hence, the study of spontaneously formed, equilibrium vesicles has recently been done intensively.

Recently, however, reports on spontaneous vesicle formation have been seen in some mixed surfactant systems. Vesicles form spontaneously in aqueous mixtures of dodecyl dimethylammonium hydroxide (DDAOH) or acetate (DDAA) and dodecyl dimethylammonium bromide (12), and alkyldimethylammnonium oxide and alcohol (13). Kaler and co-workers have reported that equilibrium vesicles can be formed spontaneously in aqueous mixtures of single-tailed anionic and cationic surfactants (e.g., cetyltrimethylammonium tosylate/ sodium dodecylbenzensulfonate (CTAT/ SDBS) $(9,14)$. They also observed spontaneously formed vesicles in mixtures of dodecyltrimethylammonium bromide/ sodium dodecyl sulfate (DTAB/ SDS) (15), and cetyltrimetylammonium bromide/ sodium octyl sulfate (CTAB/ SOS) (16) systems.

An understanding of the mechanism of vesicle to micelle transition is important in both the science and technology of surfactants. Salker et al. have shown the mechanism of temperature-dependent transition from vesicles to micelles in solutions of cetyltrimethylammonium hydroxynaphthalenecarboxylate (CTAHNC) (17). An example of the detailed studies of the transition is the case of mixed lipid- surfactant systems $(18,19)$. The transition occurred gradually with increase in the surfactant composition, from large uni-lamellar vesicles (LUV) to micelles via a small uni-lamellar vesicle (SUV) / micelles coexisting phase. The observation was explained theoretically in terms of the packing parameter favoring formation of either vesicles or micelles. The lipids have two hydrocarbon chains favoring formation of vesicles, while surfactants having a single chain tend to form micelles. The mixtures of lipid and surfactant then exhibit a transition from vesicles to micelles depending on the mixing ratio. However, in aqueous mixtures of cationic and anionic surfactants, unlike in mixtures of lipids-surfactants, vesicles formed at low total concentrations transform to micelles at high total concentrations even at the fixed composition.

In the present study, we have chosen a mixed system of cetyltrimethylammonium bromide and sodium octyl sulfate, in which vesicles of an excellent dispersion stability form, and examined the cause of concentrationdependent vesicle- micelle transition in the system by means of differential interference optical microscopy, freeze-fracture electron microscopy, static and dynamic light scattering measurements, electroconductivity measurements, and FT-IR adsorption measurements.

\section{Experimental}

Cetyltrimethylammonium bromide (CTAB, Aldrich) and sodium octyl sulfate (SOS, Aldrich) were used as cationic and anionic surfactants, respectively. Distilled water for injection (Ohtsuka Pharm. Co.) was used as the solvent.

CTAB and SOS solutions at desired concentrations were prepared. These surfactant solutions were mixed at various ratios and the resultant solutions were gently stirred for $3 \mathrm{sec}$. with a vortex mixer to homogenize them. No other physical and mechanical external forces like ultrasonication were applied to the system. The stock solutions were equilibrated at room temperature. All measurement samples were equilibrated for 90 days. Dynamic light scattering measurements were done 90 days after solution preparation.

An inverted type optical microscope (Model IMT-2, Olympus) was used to observe the samples under transmission type differential interference.

Samples for electron microscopy were prepared by freeze-fracture replication. The freeze-replica samples were made using the apparatus (FR-7000A, Hitachi Science Co.) at $-150^{\circ} \mathrm{C}$. The fractured surface was replicated by evaporating platinum at an angle of $45^{\circ}$, followed by carbon at normal incidence to strengthen the replica. It was then placed on a 150 mesh copper grid after being washed with acetone and water and observed with a transmission electron microscope (TEM-1200EX, JEOL Co.).

The trapping efficiency of CTAB/ SOS vesicles was determined by the glucose dialysis technique. CTAB/ SOS solutions were prepared using 0.28 M D-(+)-glucose/ $0.02 \mathrm{M} \mathrm{NaBr}$ aqueous solution instead of $0.02 \mathrm{M}$ $\mathrm{NaBr}$ aqueous solutions as the solvent. The sample placed into a cellulose tube (Viskase Co., Inc.), through which solutes with molecular weight less than 13000 can permeate. The unencapsulated glucose was removed by dialysis against $0.16 \mathrm{M} \mathrm{NaBr}$ aqueous solu- 
tion for $12 \mathrm{~h}$. Vesicles inside the tube were disrupted by addition of ethanol, and then the amount of glucose left in the tube was determined by the mutarotase-GOD method (20).

The intensity of light scattered from the sample in a quartz optical cell was measured with a scattered light measuring apparatus (Submicron Particle Analyzer Model 4700, Malvern) equipped with a He-Ne Laser as the light source at $633 \mathrm{~nm}$ (wave length ) and $90^{\circ}$ (scattering angle).

Particle size measurements were performed with a light scattering apparatus (Model NICOMP 370, Particle Sizing System). The light source was an argon laser (light source wavelength $514.5 \mathrm{~nm}$ ) and the scattering angle was $90^{\circ}$. The particle size was autocalculated by the method of NICOMP distribution analysis.

The specific conductivity measurements were done at $25^{\circ} \mathrm{C}$ with an electroconductance meter (Model CM40s, Toa Dempa Co.) using a pair of conductance electrodes (CG-511C, Toa Dempa Co.). Readings $15 \mathrm{~min}$ after the start of measurement were taken as the measured conductivities.

A FT-IR spectrometer (MAGNA-IR560, Nicolet Instrument Co.) was used to measure IR adsorption spectrum. Measurements were carried out at $22 \pm 1{ }^{\circ} \mathrm{C}$ by the ATR method using a zinc selenide ( $\mathrm{ZnSe})$ cell with $0.5 \mathrm{~cm}^{-1}$ resolution and 264 integrations. The Happ- Genzel function was used for apodization.

\section{Results and Discussion}

\section{$3 \cdot 1$ Phase Behavior of CTAB/ SOS Mixed Solutions}

The phase state was observed by optical and transition electron microscopy for CTAB/ SOS mixed solutions prepared at various compositions and concentrations and a ternary phase diagram was constructed for the CTAB/ SOS/ $\mathrm{H}_{2} \mathrm{O}$ system. Figure 1 is the phase diagram thus obtained.

The solution was bluish (Fig. 2) in the region indicated by $\mathrm{V}$ in the Fig. 1, Fig. $\mathbf{3 a}$ is an image of a freezefracture replica of the solution in this region where unilamellar vesicles (LUV) with a diameter of approximately $100 \mathrm{~nm}$ were observed. This microscopic image was quite similar to those of vesicles so far reported (14) and, trapping efficiency measurements of these vesicles clearly indicated that they have an inner aqueous phase. The region was then designated as the vesi-

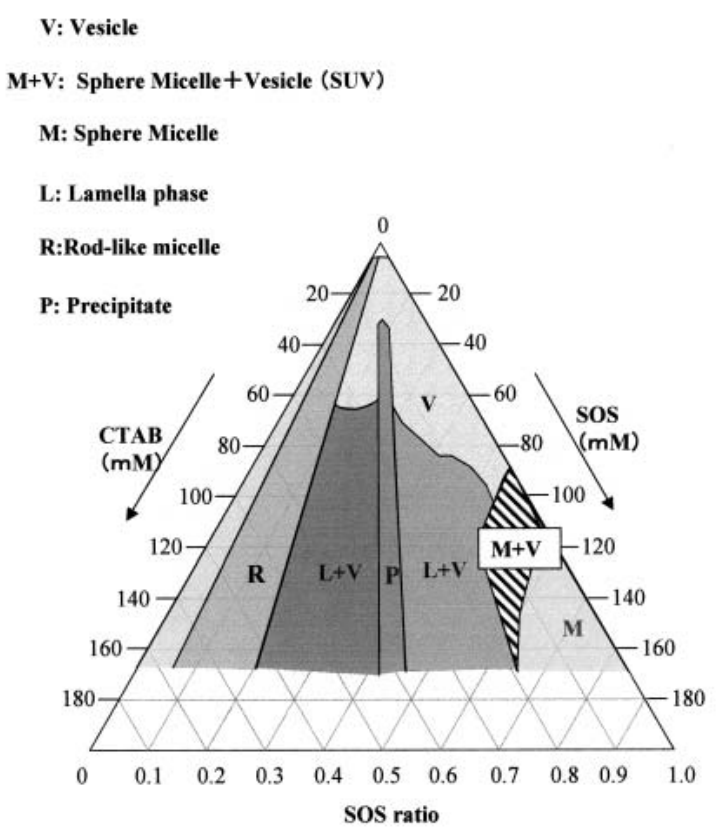

Fig. 1 Ternary Phase Diagram of CTAB/ $\mathrm{SOS} / \mathrm{H}_{2} \mathrm{O}$ System.

cles ( $\mathrm{V}$ phase) region.

In the region $\mathrm{M}$, solutions were isotropic and the size of assemblies formed was approximately $5 \mathrm{~nm}$. In addition, these solutions had no trapping ability. This region was thus inferred to be the mixed micelle (M phase) region.

In the region between the $\mathrm{V}$ and $\mathrm{M}$ phases (shaded region), the solution was isotropic and dynamic light scattering measurements showed that the size distribution of assemblies formed in this region has two peaks at about 5 and $30 \mathrm{~nm}$, respectively. The peaks around 5 $\mathrm{nm}$ can be ascribed to micelles judging from the small size. Molecular assemblies at around $30 \mathrm{~nm}$ are likely to be vesicles since the solution had $0.75 \%$ trapping efficiency for glucose. A freeze fracture TEM image of the solution in this region (Fig. 3b) also showed the for-

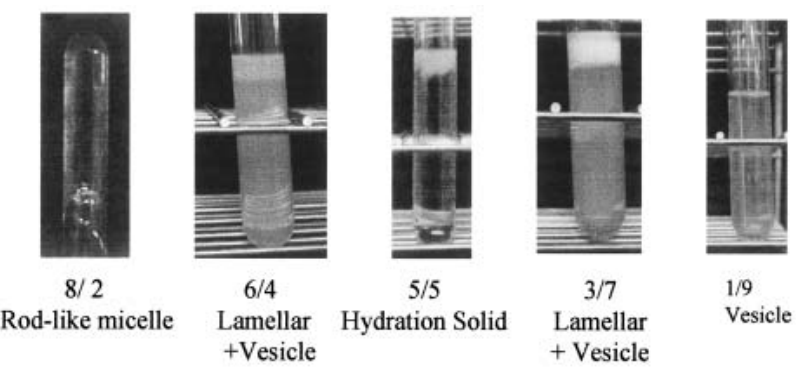

Fig. 2 Visual Observations of CTAB/ SOS/ $\mathrm{H}_{2} \mathrm{O}(100 \mathrm{mM})$ System. 

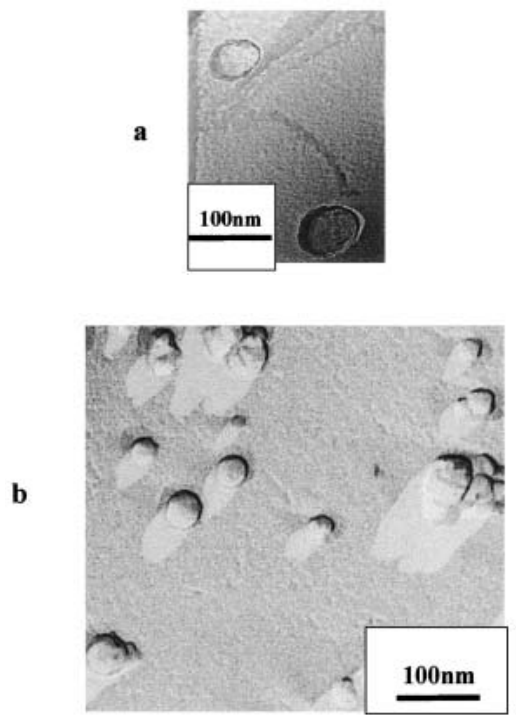

Fig. 3 TEM Image of (a) Freeze- Fractured SOS-Rich Vesicle Fraction $(\mathrm{CTAB} / \mathrm{SOS}=1 / 9,80 \mathrm{mM})$ and (b) the SOS-Rich Small Uni Lamellar Vesicle Fraction $(\mathrm{CTAB} / \mathrm{SOS}=1 / 9,100 \mathrm{mM})$.

mation of small unilamellar vesicles (SUV). Hence, we identified this region as the small unilamellar vesicle and micelle $(\mathrm{V}+\mathrm{M})$ coexisting phase. This is a novel finding on the phase behavior of aqueous "catanionic" system.

The $\mathrm{L}+\mathrm{V}$ region was the one in which a turbid top (lamellar) phase and a birefringent bottom (vesicular) phase coexist in equilibrium (Fig. 2). A typical optical micrograph showed Maltese-crosses in the top phase (Fig. 4). The trapping efficiency of the bottom phase was $6.2 \%$, suggesting vesicle formation in it.

The region $\mathrm{R}$ was found to be the rod-like micelle region because the solution was highly viscous and did not flow even when the test tube containing it was turned upside down (Fig. 2). Mixtures of cationic and

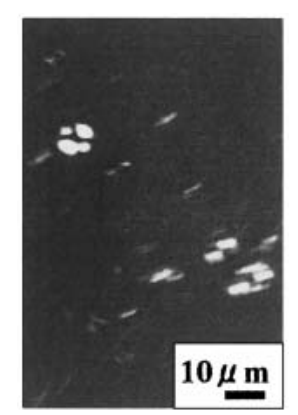

Fig. 4 Optical Micrograph in Dark Field for Lamellar Phase Fraction $(\mathrm{CTAB} / \mathrm{SOS}=4.5 / 5.5120 \mathrm{mM})$. anionic surfactant solutions, such as CTAT / SDBS system, form rod-like micelles (21). The rod-like micelle formation was brought about by a decreased electrostatic repulsion between the headgroups of respective cationic and anionic surfactants. Hence, the region was designated as the rod-like micelle phase.

Precipitates were observed at around the equimolar surfactant mixing ratio in the region $\mathrm{P}$ (Fig. 2). ${ }^{1} \mathrm{H}$ NMR analysis indicated that the precipitates (Fig. 5) contain both CTAB and SOS, suggesting the formation of a pseudo-double-tailed complex of CTAB and SOS in solution.

The ternary phase diagram showed that vesicles formed in the anion (SOS) - rich region at low total concentrations transform to micelles at high concentrations through their coexisting phase.

\subsection{Vesicle (V) - Micelle/ Small Unilamellar Vesicle $(\mathbf{M}+\mathbf{V})$ Transition}

Figure 6 shows the relationship between the intensity of scattered light and the total surfactant concentration in CTAB/ SOS mixed solutions obtained at molar ratios of $0.5 / 9.5,1 / 9,1.5 / 8.5$ and $2 / 8$. The intensity of scattered light steeply increased with increasing concentration in the vesicle region in Fig. 1. With further increase in concentration, the intensity was observed to decrease abruptly and the solution became transparent in the micelles/ small unilamellar vesicle $(\mathrm{M}+\mathrm{V})$ coexisting phase. With concentration increase beyond this region, the intensity remained constant in the single micelle region $(\mathrm{M})$, despite much further increase in

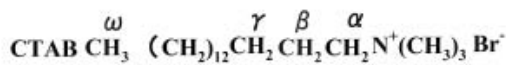

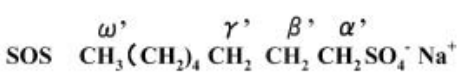

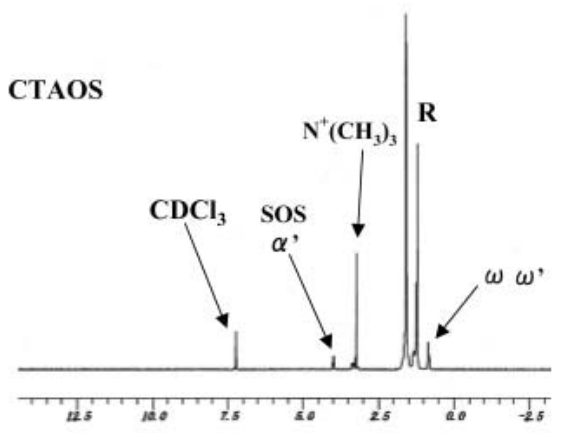

Fig. $5 \quad{ }^{1} \mathrm{H}$ NMR Spectrum of Cetyltrimethylammonium Octyl Sulfate, CTAOS. 


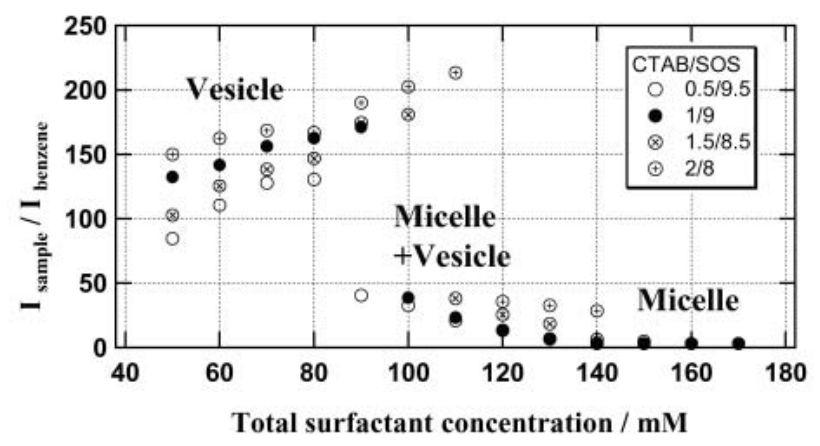

Fig. 6 Apparent Scattered Light Intensities for Aqueous Mixtures of CTAB/ SOS. concentration. Abrupt decrease in the intensity of scattered light occurred at 120,110,100 and $90 \mathrm{mM}$ at mixing ratios of $2 / 8,1.5 / 8.5,1 / 9$ and $0.5 / 9.5$, respectively.

Figures 7 and 8 show the relationship between the particle size of molecular assemblies and the total surfactant concentration in CTAB/ SOS mixed solutions with a $0.5 / 9.5$ composition 15 and 90 days after preparation, respectively. At low concentrations where vesicles were observed, the particle size distribution had two peaks at about $70 \sim 80$ and $200 \sim 250 \mathrm{~nm} 15$ days after preparation. The volume ratio of larger particles at $200 \sim 250 \mathrm{~nm}$ (vesicle) was higher than that of
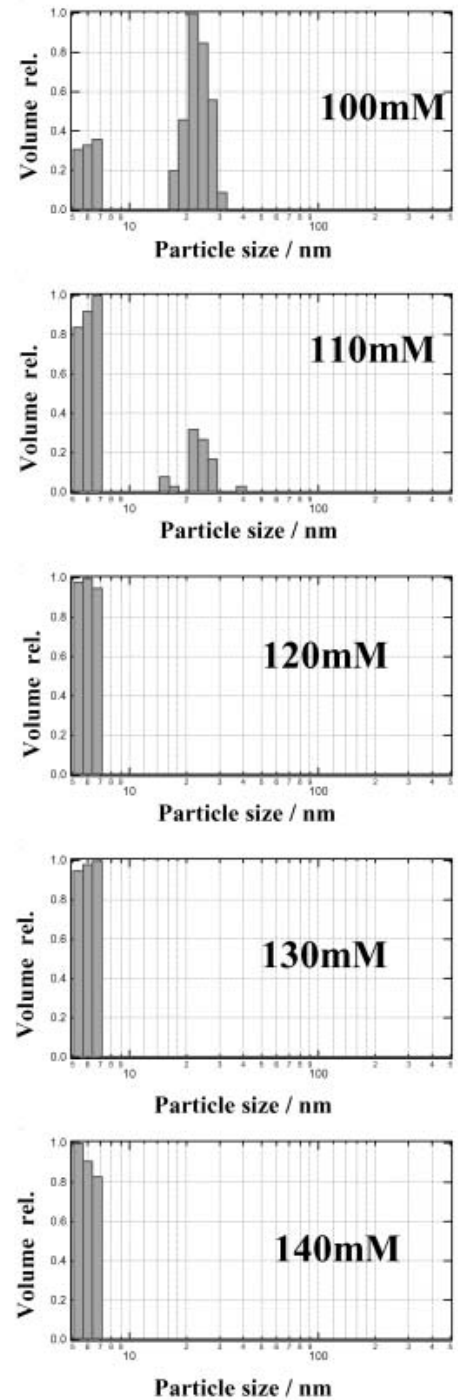

Fig. 7 Diameter Distribution of Molecular Assemblies Formed in Aqueous Mixtures of CTAB/ SOS (0.5/ 9.5, 15 days after Preparation). 
smaller particles at approximately $70 \sim 80 \mathrm{~nm}$. However, two distributions merged to one with a peak at about 180- $230 \mathrm{~nm}$ (corresponding to vesicles) 90 days after preparation (Fig. 8). These results could be understood $(14,16)$ by considering that the composition of initial nonequilibrium vesicles would be quite different from the bulk surfactant composition, and hence the initial vesicle size increases to attain an optimal curvature.

In the micelle / small unilamellar vesicle $(\mathrm{M}+\mathrm{V})$ coexisting region, the particle size distribution had peaks at about 5 (micelles) and $20 \sim 30 \mathrm{~nm}$ (small unilamellar vesicles). The particle size slightly decreased at higher concentrations and remained constant even after 90 days. In addition, the size of pure micelles formed at higher concentrations was $5 \mathrm{~nm}$ in the single micelle region and independent of concentra-
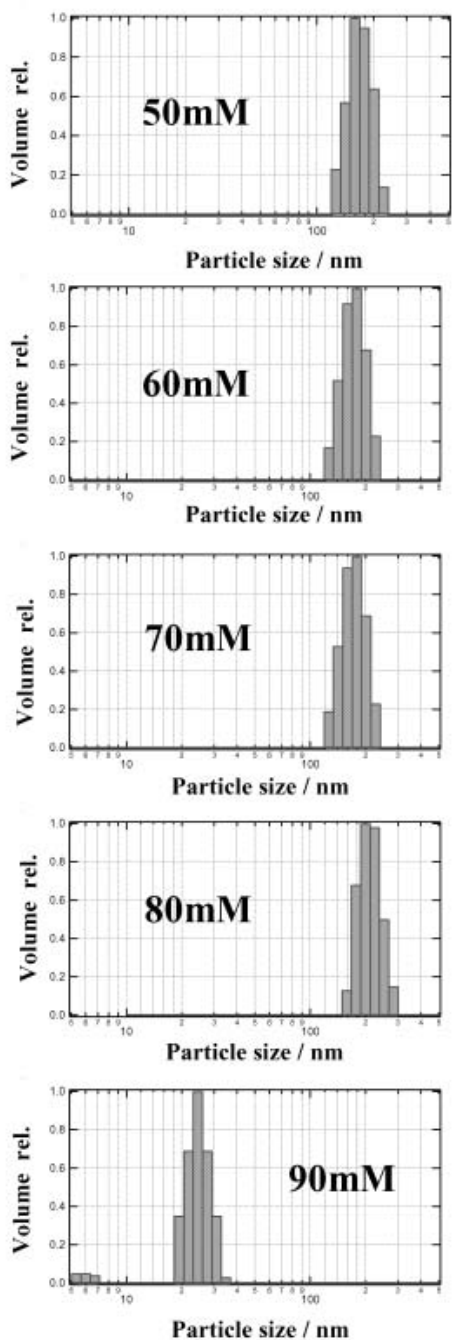

tion.

Figure 9 shows the the specific conductivity of CTAB/SOS mixed solution as a function of the total surfactant concentration. Generally, the conductivity of ionic surfactant shows a bending point at the $\mathrm{CMC}$ due to a sudden increase in counter ion binding of the surfactant above this concentration $(22,23)$. The conductivity of the mixed solution exhibited a bending point at the same concentration as that where the vesicle (V)/ micelle + vesicle $(\mathrm{M}+\mathrm{V})$ transition was observed in static light scattering and particle size measurements as seen in the Fig. 6 and 7. The concentrations at the bending points were 120,100 , and $90 \mathrm{mM}$ at CTAB/SOS mixing ratios of $2 / 8,1 / 9$, and $0.5 / 9.5$, respectively.

Figure 10 shows the relation between the wavenumber for the asymmetric stretching vibration of $\mathrm{SO}_{3}{ }^{-}$in
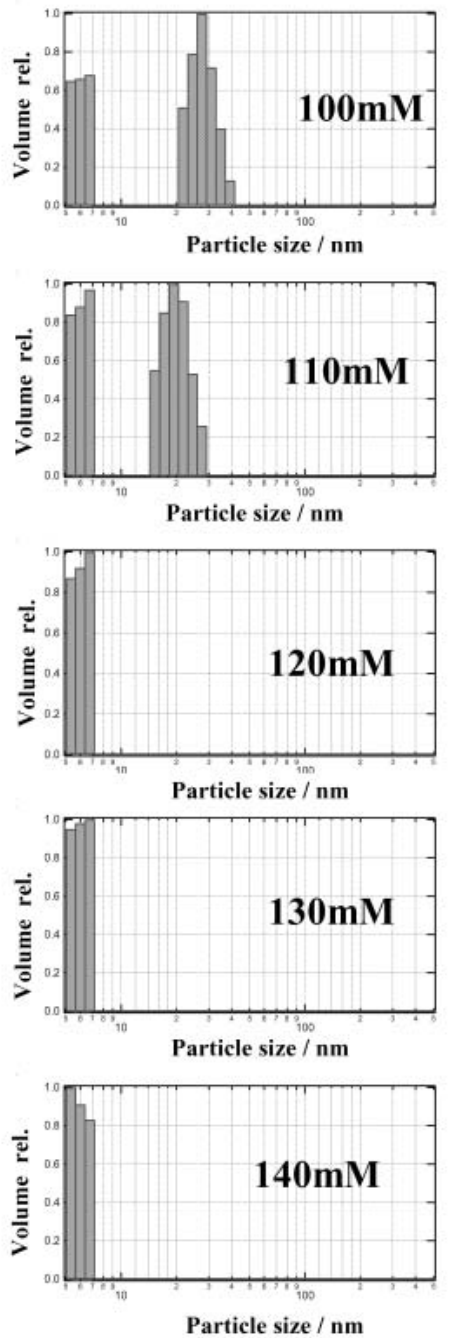

Fig. 8 Diameter Distribution of Molecular Assemblies Formed in Aqueous Mixtures of CTAB/ SOS (0.5/ 9.5, 90 days after Preparation). 


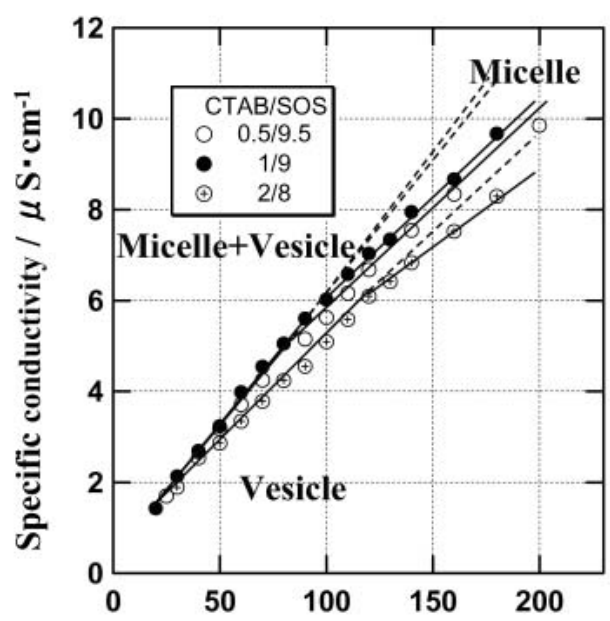

Total Surfactant Concentration / mM

Fig. 9 Specific Conductivities of Aqueous Mixtures of CTAB/ SOS as a Function of Total Surfactant Concentration.

SOS solution and concentration. The wavenumber shifted to the lower wavenumber side when SOS molecules changed their state from monomers to micelles at the CMC $(120 \mathrm{mM})$. The wavenumber remained constant in the monomer region while it decreased with increasing concentration in the micelle region. The shift in the IR absorption is caused by the binding of the counterions of $\mathrm{SO}_{3}{ }^{-}$groups to the surface of micelles to alter the surface charge (23). The reduced charges are redistributed among the $\mathrm{S}$ and $\mathrm{O}$ atoms, thereby changing the dipole moment of the group to cause the IR absorption peak to shift to the lower wave number side.

Figures 11 and 12 respectively show the relationship between the wavenumber and concentration for CTAB/ SOS mixed solution at mixing ratios of $1 / 9$ and $2 / 8$. The transition from vesicles $(\mathrm{V})$ to micelle- vesicle $(\mathrm{M}+$

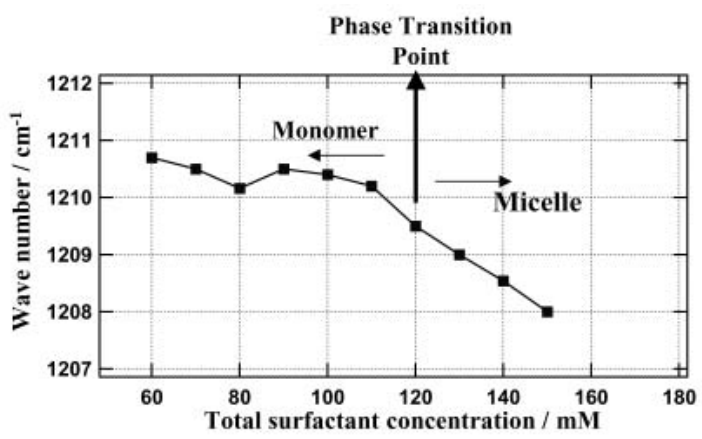

Fig. 10 Concentration Dependence of the $v_{\mathrm{a}}\left(\mathrm{SO}_{4}{ }^{-}\right)$ Wavenumber of Aqueous SOS Solution.
V) caused the wavenumber to shift downward. The wavenumber remained unchanged independently of counterion in the vesicle region, whereas it reduced with rising concentration. The above results suggest that the charge density per unit surface area of molecular assemblies is higher for micelles than that for vesicles, that is, the major constituent of vesicles is CTABSOS complex while micelles contain excess SOS molecules.

\subsection{The Mechanism of Vesicle to Micelle Transition}

In aqueous mixed solutions of cationic and anionic surfactants, a pseudo double-tailed surfactant is formed as a result of the electrostatic interaction between positive and negative charges on the hydrophilic groups of the surfactants and vesicles formed when the complex surfactant has a molecular structure favorable to bimolecular membrane formation (6).

Table 1 lists the vesicle $(\mathrm{V}) /$ micelle + small vesicle $(\mathrm{M}+\mathrm{V})$ phase transition concentrations at different mixing ratios in the SOS-rich region. The table also

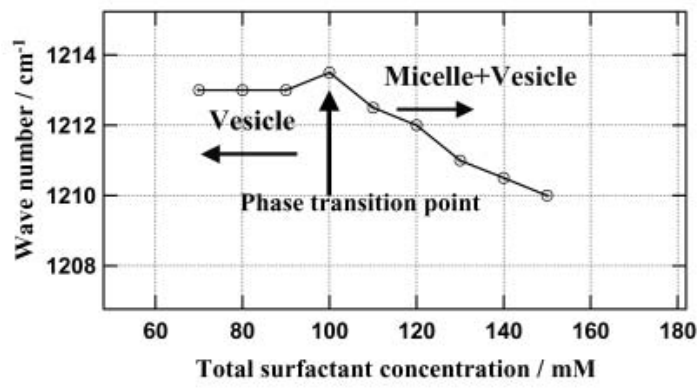

Fig. 11 Concentration Dependence of the $v_{\mathrm{a}}\left(\mathrm{SO}_{4}{ }^{-}\right)$ Wavenumber of Aqueous CTAB/ SOS $=1 / 9$ Solution.

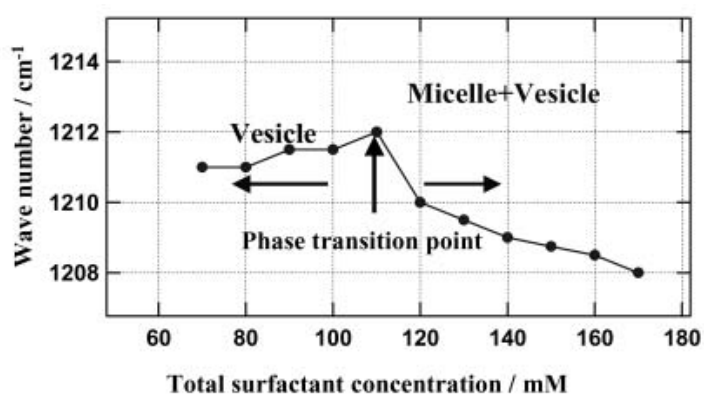

Fig. 12 Concentration Dependence of the $v_{\mathrm{a}}\left(\mathrm{SO}_{4}{ }^{-}\right)$ Wavenumber of Aqueous CTAB/ SOS $=2 / 8$ Solution. 
gives the total surfactant concentrations at which the phase transition occurs, (1) SOS concentrations at respective phase transition concentrations, and (2) excess SOS concentrations when all CTAB molecules are assumed to bind to SOS molecules to form pseudo double-tailed surfactant molecules. At the respective phase transition concentrations, SOS concentration (2) was somewhat lower than the CMC of SOS (120 mM).

Furthermore, a bending point was observed at the vesicle $(\mathrm{V})$ - micelle + small vesicle $(\mathrm{M}+\mathrm{V})$ transition on the conductivity- concentration plots. The downward shift in the wavenumber of IR absorption (ascribed to the asymmetrical stretching of $\mathrm{SO}_{3}{ }^{-}$) was observed at the phase transition concentration. The above findings suggest that the vesicle $(\mathrm{V})$ - micelle + small vesicle $(\mathrm{M}+\mathrm{V})$ transition is due to the micelle formation by excess SOS molecules which are not involved in the complex formation with CTAB.

Vesicles (V) - micelles (M) transition in mixtures of cationic and anionic surfactants is similar to that found in mixtures of lipid- surfactant systems (17-19). These systems gradually changes from, [1] single vesicle region $(\mathrm{V}),[2]$ micelle/ small vesicle coexist region $(\mathrm{M}+\mathrm{V}),[3]$ single micelle $(\mathrm{M})$ region. The particle size in the single vesicle region [1] increased depending on the surfactant concentration. In the region (2) micelle/ small vesicle coexisting region $(\mathrm{M}+\mathrm{V})$, small vesicles and micelles which solubilized lipids exist. In the single micelle region [3], the complex molecules are solubilized.

The above view would lead to a mechanism for the vesicle - micelle transition in our system that pseudo

Table 1 The Phase Transition Concentration (Total Surfactant, (1) SOS, and Excess SOS which does not Form the Pseudo Double-Tailed Surfactant) from Vesicles $(\mathrm{V})$ to Micelles + Small UniLamella Vesicle $(\mathrm{M}+\mathrm{V})$.

\begin{tabular}{cccc}
\hline CTAB/ SOS & $\begin{array}{c}\text { Total surfactant } \\
\text { concentration } \\
\text { CTAB+SOS (mM) }\end{array}$ & (1) & (2) \\
\hline $0.5 / 9.5$ & 90 & 89 & 81 \\
$1 / 9$ & 100 & 90 & 80 \\
$1.5 / 8.5$ & 110 & 94 & 77 \\
$2 / 8$ & 120 & 96 & 72 \\
\hline (1) Concentration of SOS & & \\
(2) Concentration of excess SOS is not involved in pseudo double- \\
tailed surfactant (CTAOS) formation
\end{tabular}

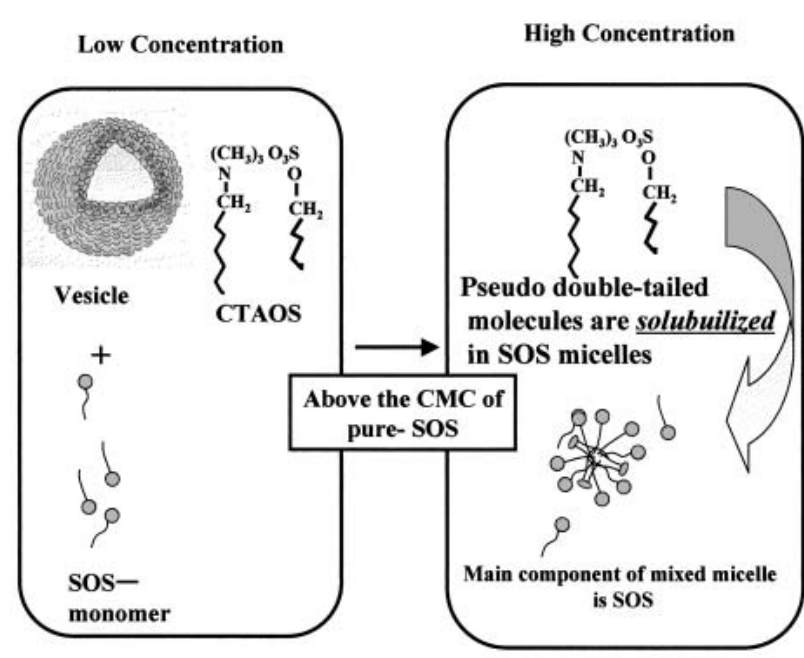

Fig. 13 Model for the Vesicle - Micelle Transition of $\mathrm{CTAB} / \mathrm{SOS} / \mathrm{H}_{2} \mathrm{O}$ System.

double- tailed surfactant molecules (CTAOS) which constitute vesicles at low concentrations are solubilized in SOS micelles at high concentrations where free SOS molecules alone can form micelles (Fig. 13). The CMC of surfactant generally lowers in the presence of hydrophobic substances. The vesicle- micelle transition was observed at concentrations of excess SOS not forming pseudo- double tailed surfactant somewhat lower than the CMC of pure SOS. The higher was the proportion of highly hydrophobic pseudo double-tailed surfactant molecules, the lower was the transition concentration. This would indicate that a larger amount of pseudo double- tailed surfactant is solubilized by micelles formed by excess SOS molecules.

\section{Conclusions}

In the $\mathrm{CTAB} / \mathrm{SOS}$ catanionic system, vesicles (V) observed at low concentration transformed to micelles (M) through micelle/ small vesicle $(\mathrm{M}+\mathrm{V})$ coexisting region - single micelles $(\mathrm{M})$ region. The phase transition from vesicles $(\mathrm{V})$ to micelles/ small vesicles $(\mathrm{M}+$ $\mathrm{V}$ ) took place at the concentration around the $\mathrm{CMC}$ of pure-SOS in the aqueous mixed solutions. Micelles formed at high concentrations were found to bind the counterions more tightly than vesicles formed at low concentrations. These findings imply that the vesiclemicelle transition was brought about through the solubilization of pseudo double- tailed surfactant molecules into micelles formed by free SOS molecules present in the bulk. 


\section{References}

1. M. ROSEN, J. Surfactant and Interfacical Phenomena, 2nd ed., Wiley-Interscience (1965).

2. D. OHLENDORF and W. INTERTHAL, in Solution Behavior of Surfactants, New York, 1589-1595 (1980).

3. M.J. OSTRO, Liposomes, Marcel Dekker Inc. (1987).

4. S. BHANDARKAR and A. BOSE, Synthesis of Submicrometer Crystals of Aluminum Oxide by Aqueous Intravesicular Precipitation, J. Colloid Interface Sci., Vol. 135, 531-538 (1990).

5. J. FENDLER, Membrane Mimetic Chemistry, Springer-Verlog (1983).

6. R.A. SALKAR, D. MUKESH, S.D. SAMANT and C. MANOHAE, Mechanism of Micelle to Vesicle Transition in Cationic-Anionic Surfactant Mixtures, Langmuir, Vol. 14, 3778 3782 (1998).

7. H.W. HOYER, A. MARMO and M. ZOELLER, On the Molecular Basis of Some Current Theories of Diffusion, J. Phys. Chem., Vol. 65, 1804-1806 (1961).

8. P.M. HOLLAND and D.N. RUBINGH, Nonideal Multicomponent Mixed Micelle Model, J. Phys. Chem., Vol. 87, 1984-1990 (1983).

9. E.W. KALER, A.K. MURTHY, B.E. RODRIGUEZ and J.A.N. ZASADZISKI, Spontaneous Vesicle Formation in Aqueous Mixtures of Single- Tailed Surfactants, Science, Vol. 92, 66716698 (1992).

10. O. SODERMAN, K.L. HERRINGTON, E.W. KALER and D.D. MILLER, Transition from Micelles to Vesicles in Aqueous Mixtures of Anionic and Cationic Surfactants, Langmuir, Vol. 13, 5531-5538 (1997).

11. H. IMAMURA, K. TSUCHIYA, Y. KONDO, N. YOSHINO, T. OHKUBO, H. SAKAI and M. ABE, Phase Behavior of Mixed Solutions of a Cationic Surfactant with a Ferrocenyl Group and an Anionic Surfactant, J. Oleo Sci., Vol. 54, 125-134 (2005).

12. D.D. MILLER, L.J. MAGID, D.F. EVANS, Fluorescence Quenching in Double-Chained Surfactants. 2. Experimental Results, J. Phys. Chem., Vol. 94, 5921-5930 (1990).

13. H. HOFFMANN, U. MUNKWRT, H.W. MEYER and W. RICHTHER, From Vesicles to the L3 (sponge) Phase in Alkyldimethylamine Oxide/ Heptanol Systems, Langmuir, Vol. 8, 2629-2638 (1992).

14. E.W. KALER, K.L. HERRINGTON, A.K. MURTHY and J.A.N. ZASADZISKI, Phase Behavior and Structures of Mix- tures of Anionic and Cationic Surfactants, J. Phys. Chem., Vol. 92, 6698-6707 (1992).

15. K.L. HERRINGTON, E.W. KALER, D.D. MILLER, J.A.N. ZASADZINSKI and S.CHIRUVOLU, Phase Behavior of Aqueous Mixtures of Dodecyltrimethylammonium Bromide (DTAB) and Sodium Dodecyl Sulfate (SDS), J. Phys. Chem., Vol. 97, 13792-13802 (1993).

16. M.T. YATICILLA, K.L. HERRINGTON, L.L. BRASHER, E.W. KALAR and J.A.N. ZASADZINSKI, Phase Behavior of Aqueous Mixtures of Cetyltrimethylammonium Bromide (CTAB) and Sodium Octyl Sulfate (SOS), J. Phys. Chem., Vol. 100, $5874-$ 5879 (1996).

17. S. ALMAG, T. KUSHINIR, R. NIR and D. LICHTENBERG, Kinetics and Structural Aspects of Reconstitution of Phosphatidylcholine Vesicles by Dilution of PhosphatidylcholineSodium Cholate Mixed Micelles, Biochemistry, Vol. 25, 25972605 (1986).

18. S. ALMG, B.J. LiTMAN, W. WIMELY, J. COHEN, E.J. WACHTEL, Y. BAEWNHOLZ, A. BEN- SHAUL and D. LICHTENBERG, States of Aggregation and Phase Transformations in Mixtures of Phosphatidylcholine and Octyl Glucoside, Biochemistry, Vol. 29, 4582-4592 (1990).

19. K. HORBASCHEK, H. HOFFMAN, HOFFMANN and C. THUNING, Formation and Properties of Lamellar Phases in Systems of Cationic Surfactants and Hydroxy-Naphthoate, $J$. Colloid and Interface Sci., Vol. 206, 439-441 (1998).

20. W.R. BRODE, J.H. GOULD and G.M. WYMAN, The Relation between the Absorption Spectra and the Chemical Constitution of Dyes. XXV. Phototropism and cis-trans Isomerism in Aromatic Azo Compounds, J. Am. Chem. Soc., Vol. 74, 4641-4646 (1952).

21. R.D. KOEHLER, S.R. RAGHAVAN and E.W. KALER, Microstructure and Dynamics of Wormlike Micellar Solutions Formed by Mixing Cationic and Anionic Surfactants, J. Phys. Chem. B, Vol. 104, 11035-11044 (2000).

22. C. BORTE, V.L. CRESCENZI and A. MELE, A Study on Micelle Formation in Colloidal Electrolyte Solutions, J. Phys. Chem., Vol. 63, 650-653 (1959).

23. A.W. RALSTON, C.W. HOERR and E.J. HOFFMANN, Studies on High Molecular Weight Aliphatic Amines and their Salts. IV. Electrical Conductivities of Aqueous Solutions of the Hydrochlorides and Acetates of Dodecyl-and Octadecylamines, J. Am. Chem. Soc., Vol. 64, 97-101 (1942). 